\title{
Controlling Uncertainty in Personal Positioning at Minimal Measurement Cost
}

\author{
Hui Fang ${ }^{1}$, Wen-Jing Hsu ${ }^{1}$, and Larry Rudolph ${ }^{2}$ \\ 1 Nanyang Technological University \\ Nanyang Avenue, Singapore 639798 \\ fang0025@ntu.edu.sg, hsu@pmail.ntu.edu.sg \\ 2 Massachusetts Institute of Technology \\ 77 Massachusetts Avenue, Cambridge, MA 02139, USA \\ rudolph@csail.mit.edu
}

\begin{abstract}
One interesting scenario in personal positioning involves an energy-conscious mobile user who tries to obtain estimates about his positions with sufficiently high confidence while consuming as little battery energy as possible. Besides obtaining estimates directly from a position measuring device, the user can rely on extrapolative calculations based on a user movement model and a known initial estimate. Because each measuring probe usually incurs a substantially higher cost than the extrapolative calculation, the objective is to minimize the overall cost of the measurement probes.

Assuming that the user moves at a normally-distributed velocity, we consider two scenarios which differ in the probing devices used. In the first scenario, only one probing device is used. In this case, the aim is to minimize the total number of probes required. In the second scenario, two types of positioning devices are given, where one type of devices offers a higher positioning precision, but also at a greater probing cost. In this case, the aim is to choose an optimal combination of probes from the two types of devices.

For both scenarios, we present algorithms for determining the minimumcost probing sequences. The algorithms are computationally efficient in reducing the searching space of all possible probing sequences. Our approach is based on Kalman Filtering theory which allows to integrate estimates obtained from the measurements and the extrapolative calculations. The variances in the estimates can provably stay below the specified level throughout the journey.

To the best of our knowledge, these results appear to be the first that uses a mathematically rigorous approach to minimize the probing cost while guaranteeing the quality of estimates in personal positioning.
\end{abstract}

\section{Introduction}

With the fast growth of personal mobile devices, people are developing diverse methods to provide efficient and accurate positioning services which are useful in many potentially interesting position-based applications, see e.g. Bhattachary and Das [2] or D'Roza et al. [4]. 
Personal positioning refers to obtaining an estimate of a mobile user's actual position at a given point in time by using individualized means. Such estimates are usually obtained either from measurements by using a positioning device such as GPS (Global Positioning System), or, alternatively, through calculations based on a predictive model of the user's movements, stretching from an existing estimate.

Naturally, due to inherent non-determinism in the user movements, the extrapolative calculations accrue uncertainty over time, and a new probe will be needed to control the uncertainty. Now because each measuring probe usually incurs a substantially higher cost than the extrapolative calculation, the objective is to minimize the overall cost of the measurement probes over a journey while keeping the uncertainties in any estimate below an acceptable level.

Although there are already many positioning algorithms based on GPS [ 5 , $4,8,1]$ or cell ID of the base stations for mobile phones $[2,14,13]$ separately, there are very few existing results that combine positioning information obtained from multiple types of positioning devices. Most cell-based positioning methods consider only the concept of the logical positions instead of the geographical positions, see, e.g. [2]. One naturally wonders whether information from multiple sources can be combined to provide a cost-effective and consistent positioning service. Elsewhere in [12], a system in which the positioning records from multiple sources are merged for the analysis of a mobile device's motion pattern was described.

For lack of absolute knowledge about the actual position value, in this paper, a positioning estimate is represented as a Gaussian distribution whose mean is interpreted as the estimated position, and the variance as a measure of the uncertainty of the estimate. With this formulation, we are able to make use of Kalman Filter [6] to combine position estimates obtained from different sources. The problem is now reduced to finding probing sequences that will minimize the probing cost while keeping the variances ${ }^{3}$ in the estimates below a required level.

Assuming that the user moves at a normally-distributed velocity, we consider two scenarios which differ in the probing devices used. In the first scenario, only one probing device is used. In this case, the aim is to minimize the total number of probes required. In the second scenario, two types of positioning devices are given, where one type of devices offers a higher positioning precision, but also at a greater probing cost. In this case, the aim is to choose an optimal combination of probes from the two types of devices.

For both scenarios, we present computationally efficient algorithms that can determine a required optimal probing sequence. The algorithms are based on Kalman Filtering theory which allows to integrate estimates obtained from the measurements and the extrapolative calculations. The variances in the estimates can provably stay below the specified level throughout the journey.

\footnotetext{
${ }^{3}$ Equivalently, standard deviations
} 
To the best of our knowledge, these results appear to be the first that uses a mathematically rigorous approach to minimize the probing cost while guaranteeing the quality of estimates in personal positioning.

The remainder of the paper is organized as follows. Section 2 presents the formulation and notations. Our position inference method is presented in Section 3. Then we discuss how to control the variances of the inferred estimates in Section 4. In Section 5 the optimal probing strategy with the minimum cost is achieved. Section 6 introduces the previous work related to personal positioning. Finally in Section 7 we summarize the results and conclude with open problems for future work.

\section{Definitions and assumptions}

An estimate of the device's actual position $z$ will be modeled by a 2-D Gaussian random variable written as

$$
z \sim N\left(\mu, \sigma^{2} \cdot I\right),
$$

where $\mu$ denotes its mean and $\sigma^{2} \cdot I$ denotes its covariance matrix. By assumption of Gaussian distribution, given a measurement $z$, the most likely estimate of user's actual position is $\mu$. Moreover, the variance $\sigma^{2}$ reflects how likely user's actual position is around the best estimate. The relationship between the region of high probability and the variance is given by Theorem 0 , as shown in [3].

Theorem 0. Confidence ellipse: Let $x \sim N\left(0, \sigma_{x}^{2}\right), y \sim N\left(0, \sigma_{y}^{2}\right)$, and $x, y$ be independent of each other. The probability that $(x, y)$ lies in the area $E=\left\{(x, y): \frac{x^{2}}{\sigma_{x}^{2}}+\frac{y^{2}}{\sigma_{y}^{2}} \leq r^{2}\right\}$ is given by $P=1-e^{-r^{2} / 2}$.

$E$ is called $P$-confidence ellipse, which is centered around the mean. For our case in which $\sigma_{x}=\sigma_{y}=\sigma$, the ellipse is a circle whose radius is given by $r \sigma$

where $r=\sqrt{2 \ln \frac{1}{1-P}}$. In practice, the confidence $P$ is often set to be a constant $95 \%$.

\section{Position inference by using Kalman filter}

A common scenario in personal positioning arises when the mobile user embarks on a journey at a variable velocity, and he would like to ensure that during the journey any estimate about his position can be made with a sufficiently low variance. The position estimates may be obtained by carrying out a number of measurement probes in conjunction with calculations based on the user velocity model.

Kalman filtering [15] provides a convenient framework for describing mobile user's movement model and making predictions given the measurements. Since Richard Kalman discovered it in 1960s, the Kalman filtering (KF) has been widely used in many areas such as navigation, manufacturing, and dynamical 
control etc. A Kalman filter is a recursive data processing algorithm that estimates the state of a noisy linear dynamic system. It processes all available measurements to estimate the state, including both accurate and inaccurate measurements. It uses knowledge of the system and sensor dynamics, probabilistic descriptions of the system and measurement noises, and any available data about the initial values of the state to achieve the estimate while minimizing the mean squared error between the predicted value and the measured value. Here we will adapt the formalism to suit our specific application.

Our aim is to infer the device's position at any point within a period of time T. Let $t_{i}$ denote the point in time where the $i$-th probe is carried out.

At time $t_{k}$, the predicted position estimate $x_{k}$, is given by

$$
x_{k}=x_{k-1}+\tau_{k-1} v_{k-1}+\tau_{k-1} w_{k-1},
$$

and a measurement, $z_{k}$, is given by

$$
z_{k}=x_{k}+r_{k}
$$

where $v_{k}=\left[v_{l o n}(k), v_{l a t}(k)\right]^{T}$ denotes the velocity, and $\tau_{k-1}=t_{k}-t_{k-1}$. Specifically, the velocity is obtained from the mobile user's history statistically which results in a noise $w_{k}$. The random variables $w_{k}$ and $r_{k}$ represent the velocity noise and measurement noise, respectively. They are assumed to be white noises ${ }^{4}$, normally-distributed and independent of each other.

$$
\begin{aligned}
& w_{k} \sim N\left(0, \sigma_{w}^{2} I\right), \\
& r_{k} \sim N\left(0, \sigma_{r_{k}}^{2} I\right),
\end{aligned}
$$

In practice, the standard deviation of velocity noise $\sigma_{w}$ might also change with each time step. Here, however, we assume it to be a constant.

Using Kalman filter (KF for short), we can combine two (or more) estimates that are obtained either from predictions or measurements. Given two concurrent independent estimates, the KF method can combine them to generate a new estimate; moreover, the new estimate is still Gaussian [15]. Theorem 1 states this fact more precisely.

Theorem 1 (KF-1): Let $N\left(\mu, V_{1}\right)$ and $N\left(\nu, V_{2}\right)$ be $x$ 's probability density functions (PDF) conditional on two independent estimates respectively. Then $x$ 's PDF conditional on $\mu$ and $\nu$ is a Gaussian distribution $N(\hat{x}, V)$ where

$$
\begin{gathered}
\hat{x}=\mu+Q(\nu-\mu), \\
V=V_{1}-Q \cdot V_{1},
\end{gathered}
$$

and, in (7),

$$
Q=V_{1}\left(V_{1}+V_{2}\right)^{-1}
$$

\footnotetext{
${ }^{4}$ A white noise is a random signal with a flat power spectral density, i.e., the signal's power spectral density has equal power in any band, at any center frequency, having a given bandwidth.
} 


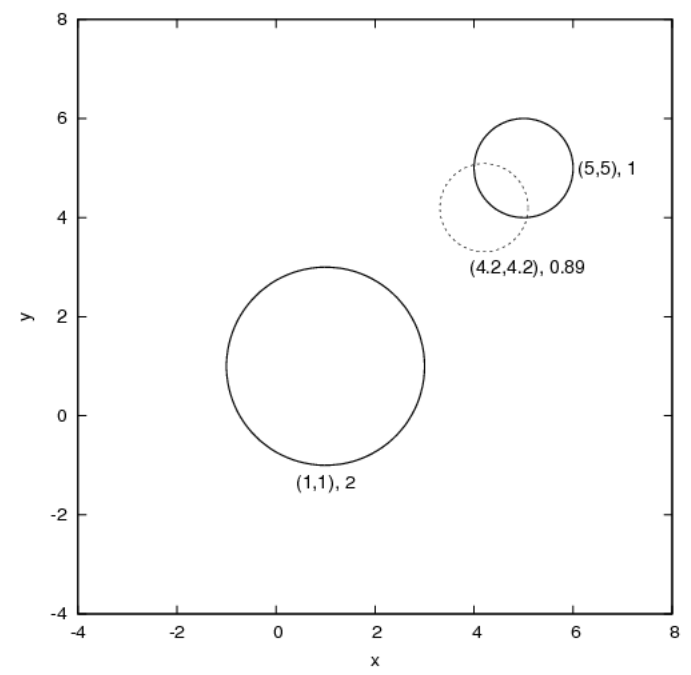

Fig. 1. The two ellipses centered at $(1,1)$ and $(5,5)$ respectively represent two concurrent measurements with the radii reflecting their standard deviations, i.e., $\sigma_{1}=2$ and $\sigma_{2}=1$. By using KF-1, a new estimate can be obtained from merging the two pieces of information, which is shown as the dotted circle, centered at $(4.2,4.2)$ with $\sigma=0.89$.

From Theorem KF-1, it can be shown that the variance of the resulting estimate will decrease after merging the two estimates, i.e., the certainty on the position estimate is improved with more position information. Specifically, Figure-1 depicts two measurements indicating positions $\mu=(1,1)$ and $\nu=(5,5)$, and their levels of uncertainty represented by the standard deviations are 2 and 1 , respectively. Given two measurements, a new estimate indicates the position $(4.2,4.2)$ with a higher certainty $(\sigma=0.89)$. The estimated position is closer to $\nu$ than to $\mu$ because the measurement $\nu$ is more certain than $\mu$.

Theorem KF-2 below says that, unless new information is obtained from measurements, the variance of an estimate derived purely from predictions will degrade over time because of variations in velocity.

Theorem 2 (KF-2): Let $N\left(x_{k}, V_{k}\right)$ be the position of the object concerned at the time $t_{k}$. Assuming the probabilistic mobility model described in (2), the position estimate $N\left(x_{k+1}, V_{k+1}\right)$ at the time $t_{k+1}$ is given by

$$
\begin{gathered}
x_{k+1}=x_{k}+\tau_{k} v_{k}, \\
V_{k+1}=V_{k}+\tau_{k} V_{w} .
\end{gathered}
$$

KF-1 and KF-2 together form the basis of our position inference method which treats both the predictions and measurements uniformly and minimizes the mean square error between prediction and measured position. The complete position estimation algorithm is summarized as follows. 
(1) Prediction update:

$$
\begin{gathered}
\hat{x}_{k}^{-}=\hat{x}_{k-1}+\tau_{k-1} v_{k-1}, \\
V_{k}^{-}=V_{k-1}+\tau_{k-1} V_{w} .
\end{gathered}
$$

(2) Measurement update:

$$
\begin{gathered}
K_{k}=V_{k}^{-}\left(V_{k}^{-}+V_{r_{k}}\right)^{-1}, \\
\hat{x}_{k}=\hat{x}_{k}^{-}+K_{k}\left(z_{k}-\hat{x}_{k}^{-}\right), \\
V_{k}=\left(I-K_{k}\right) V_{k}^{-} .
\end{gathered}
$$

\section{Analysis of probing strategies}

In this section, we will analyze several probing strategies. Here we will assume that the cost of each probe is a constant. ${ }^{5}$

The first result in Section 4.1 concerns a simple strategy where the probes are carried out periodically. We show that if the probes continue, the variance will eventually converge towards a fixed point independent of the initial estimate.

The periodic probing, however, is not optimal in terms of probing cost. Intuitively, minimizing the probing cost implies maximizing the utility of a probe. In other words, one should always delay the probe until the variance of an estimate degrades to the threshold. The second result in Section 4.2 confirms this intuition.

When two types of positioning devices are available, it turns out that all the minimum-cost strategies are equivalent to one of two generic patterns. We then prove that the optimal strategy can be found within a relatively small solution space. Section 4.3 covers the details of the algorithm to find the optimal.

\subsection{What happens if we carry out a probe periodically?}

We will show that if the same type of probes are repeated at a regular time interval, the standard deviation obtained in the positioning estimates will converge to a finite value. In order to prove this result, we firstly introduce Lemma 3 below.

Lemma 3: Let $f(x)=\frac{a}{\sqrt{1+\frac{a^{2}}{x^{2}+b^{2}}}}$, where $a, b, x \geq 0$. Then the function $y=f(x)$ has the following properties:

1. $y=f(x)$ is monotonically increasing, and $\frac{a b}{\sqrt{a^{2}+b^{2}}} \leq f(x)<a$.

2. There exists a fixed point $x^{*}=\sqrt{\frac{-b^{2}+b \sqrt{b^{2}+4 a^{2}}}{2}}$, such that $f\left(x^{*}\right)=x^{*}$

3. Let $f^{n+1}(x)=f\left(f^{n}(x)\right), f^{1}(x)=f(x)$. For any $x \geq 0, f^{n}(x) \rightarrow x^{*}$ when $n \rightarrow \infty$.

\footnotetext{
5 The energy consumption of a measurement is intricately related to many devicedependent factors such as the types of circuitry, memory access patterns, and computing capabilities etc. Here we will simply assume that a probe entails a constant amount of battery energy.
} 
Proof. The result can be obtained by calculating the first- and second-order derivatives of $f(x)$.

Figure-2 shows the function $f(x)$ mentioned in Lemma 3 . Notice that $f(\sqrt{3})=$ $\sqrt{3}$ in this example.

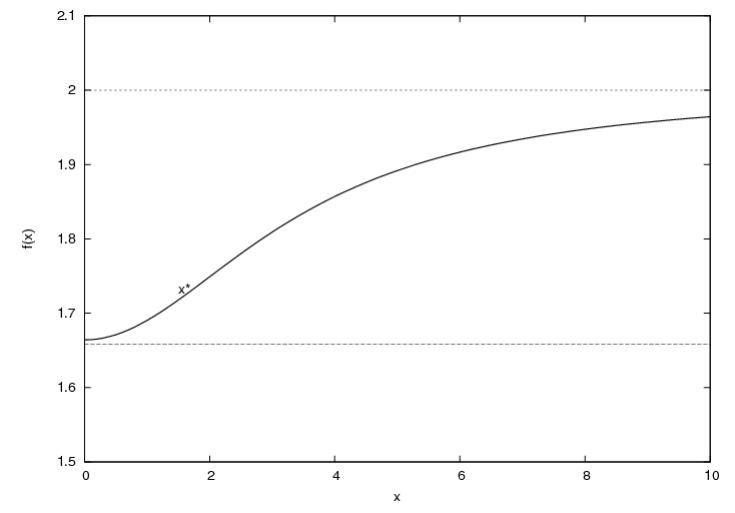

Fig. 2. The curve of function $f(x)=\frac{a}{\sqrt{1+\frac{a^{2}}{x^{2}+b^{2}}}}$ with $a=2, b=3$. Notice that $x^{*}=\sqrt{3}$ and $f(\sqrt{3})=\sqrt{3}$.

Let $\sigma$ denote the threshold value of the standard deviation required for the journey. Theorem 4 below determines the converging value of estimate of the measurements are carried out at regular intervals.

Theorem 4: Let $x_{k} \sim N\left(., \sigma_{k}^{2}\right)$ be a discrete Kalman filter process defined in Section 3. Assume that only one type of probes $\left(\sigma_{r}\right)$ is carried out at the same time interval (i.e., $\tau=t_{k}-t_{k-1}$ ). Then for any initial estimate with standard deviation $\sigma_{0}$, the standard deviation of the estimate will converge to a finite value:

$$
\lim _{k \rightarrow \infty} \sigma_{k}=\sigma^{*}\left(\tau, \sigma_{r}, \sigma_{w}\right) .
$$

Proof. Consider $x_{k} \sim N\left(., \sigma_{k}^{2}\right)$, the estimate at the time $t_{k}$, and $x_{k+1} \sim N\left(., \sigma_{k+1}^{2}\right)$, the estimate at the time $t_{k+1}$. The estimate $x_{k+1}$ is calculated based on its immediate previous estimate $x_{k}$ and the measurement $z \sim N\left(., \sigma_{r_{k+1}}^{2}\right)$ carried out at the time $t_{k+1}$. Let $\tau_{k}=t_{k+1}-t_{k}$.

According to the prediction update equations (15) and (16), the standard deviation, $\sigma_{t}$ updated periodically is given by

$$
\sigma_{t}^{2}=\sigma_{k}^{2}+\tau_{k} \sigma_{w}^{2} .
$$

Combining the latest estimate with the current measurement $z$, we have

$$
1 / \sigma_{k+1}^{2}=1 / \sigma_{t}^{2}+1 / \sigma_{r_{k+1}}^{2} .
$$




$$
\sigma_{k+1}=\frac{\sigma_{r_{k+1}}}{\sqrt{1+\frac{\sigma_{r_{k+1}}^{2}}{\sigma_{k}^{2}+\sigma_{w}^{2} \tau_{k}}}}
$$

By assumption, $\sigma_{r_{k}} \equiv \sigma_{r}, \tau_{k} \equiv \tau$. Let $a=\sigma_{r}, b=\sigma_{w} \sqrt{\tau}$. Then $\sigma_{k+1}=f\left(\sigma_{k}\right)$, where the function $f(\cdot)$ is as defined in Lemma 3. The result is immediately obtained by applying Lemma 3, which completes the proof.

Corollary 5: Let $\sigma_{k}, \sigma_{r_{k}}, \tau_{k}$, and $\sigma_{w}$ be as defined in Theorem 4. Either (1) $\sigma_{r_{k+1}} \leq \sigma_{k}$, or $(2) \tau_{k} \leq \frac{\sigma_{k}^{4}}{\sigma_{w}^{2}\left(\sigma_{r_{k+1}^{2}}^{2}-\sigma_{k}^{2}\right)}$ is a sufficient condition for $\sigma_{k+1} \leq \sigma_{k}$.

Proof. Let $\sigma_{k+1} \leq \sigma_{k}$ in the equation (20) in the proof of Theorem 4 . We have the sufficient condition

$$
\sigma_{w}^{2}\left(t_{k+1}-t_{k}\right) *\left(\sigma_{r_{k+1}}^{2}-\sigma_{k}^{2}\right) \leq \sigma_{k}^{4}
$$

If $\sigma_{r_{k+1}} \leq \sigma_{k}$, the left hand side of the inequality (21) is less than or equal to zero. Otherwise the condition (2) of Corollary 5 will imply the inequality (21) above. This completes the proof.

\section{Remarks}

Corollary 5 reflects two requirements of the new probe. Firstly, when the new probe itself has sufficiently low variance (i.e. $\phi_{i+1}<\sigma_{i}$ ), the standard deviation of the estimate at the next time step will not increase. Secondly, if the new probe does not have sufficiently low variance, then the time elapsed before the new probe must be a shorter period of time as is determined by Corollary 5 . It also suggests a strategy to choose a proper frequency to carry out new probes periodically on a journey.

Corollary 6: Let $\sigma_{k}, \sigma_{w}, \sigma_{r}=\sigma_{r_{k}}$, and $\sigma$ be as defined in Corollary 5. If probes are carried out at regular time intervals, then the probing frequency must be at least $f=\frac{\sigma_{w}^{2}\left(\sigma_{r}^{2}-\sigma^{2}\right)}{\sigma^{4}}$ such that $\sigma_{k} \leq \sigma$.

Proof. Let $\tau_{k}=1 / f, \sigma_{k}=\sigma$. From Corollary 5 we have $\sigma_{k+1} \leq \sigma_{k} \leq \sigma$. That implies all the inferred standard deviations are within the threshold. This completes the proof.

Corollary 6 implies that, using the periodic probing strategy, the ratio of frequencies of type-A probes to type-B probes should be proportional to $\frac{\sigma_{a}^{2}-\sigma^{2}}{\sigma_{b}^{2}-\sigma^{2}}$.

Figure-3 shows the standard deviation of the estimate increases over time. Without new measurements, the standard deviation will increase beyond the threshold. A probe serves to bring down the standard deviation. With periodically carried out probes, the uncertainty of the position estimates can be controlled within the required threshold. 


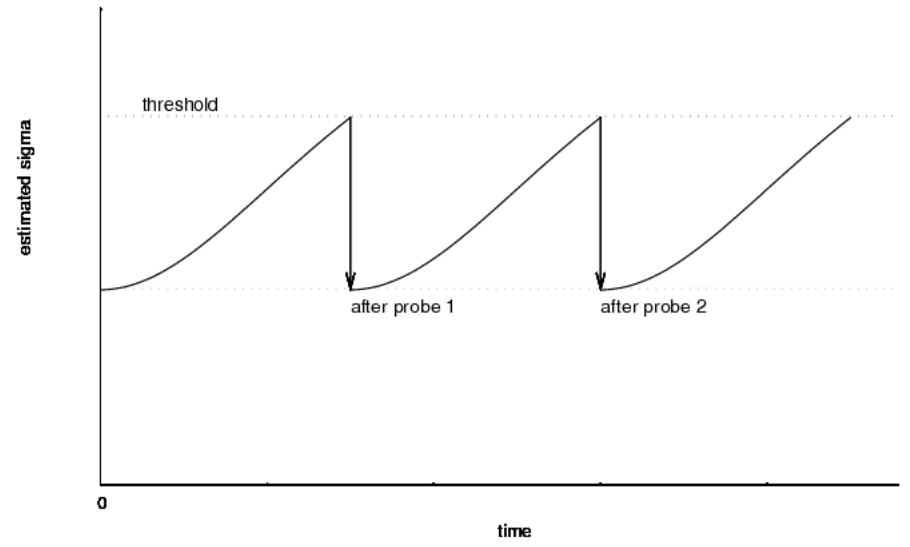

Fig. 3. The curve of the estimated $\sigma$ over time $t$. Whenever the uncertainty of the estimate increases to the threshold, a probe can bring it down.

\subsection{When is best to carry out a probe?}

For convenience, we call a time duration safe if within the period the variance of any calculated positioning estimate stays below the required threshold value. Firstly we will determine the longest safe duration with a given number of probes. Let $\sigma_{0}$ be the standard deviation of the initial estimate at $t=0$, where $\sigma_{0} \leq \sigma$. Let $\tau$ be the length of the safe duration without new probes before the standard deviation of any prediction reaches the threshold $\sigma$.

Lemma 7: By using only one probe $\sigma_{r}$ at a point of time $t \in[0, \tau]$, the safe duration is longest only when the probe is carried out at the point $t=\tau$.

Proof. By using only prediction update, at time $t$ the standard deviation is given by

$$
\sigma_{1}=\sqrt{\sigma_{0}^{2}+\sigma_{w}^{2} t} .
$$

Combining $\sigma_{1}$ with a new probe $\sigma_{r}$ by KF-1, we can obtain a new estimate with standard deviation

$$
\sigma_{2}=\sqrt{\frac{\sigma_{1}^{2} \sigma_{r}^{2}}{\sigma_{1}^{2}+\sigma_{r}^{2}}} .
$$

With the new estimate, it is possible to obtain prediction update for another duration $t_{2}$ before the estimated standard deviation degrades to the threshold $\sigma$, or, formally,

$$
\sigma^{2}=\sigma_{2}^{2}+\sigma_{w}^{2} t_{2} .
$$


So the total duration after inserting a new probe is

$$
t+t_{2}=\sigma^{2} / \sigma_{w}^{2}-\frac{\sigma_{r}^{2} / \sigma_{w}^{2}}{1+\frac{\sigma_{r}^{2}}{\sigma_{0}^{2}+\sigma_{w}^{2} t}}+t,
$$

which is a function of $t$. Calculating its derivative on $t$ shows that the overall duration monotonically increases with regard to $t$, and the maximum value is attained at the point $t=\tau$. This completes the proof.

Remarks Lemma 7 states that the longest safe duration is achieved by doing probes exactly at the end of a safe duration when the variance of the estimate based on prediction updates reaches the threshold.

Corollary 8: Given the same initial estimate, the safe duration that can be sustained via two simultaneous probes is shorter than that of two serial probes.

Proof. According to Lemma 7, the maximum valid duration is achieved only when the new probe is carried out at the end of the first probe's safe duration.

Corollary 8 implies that, to maximize the utility of a given number of probes, carrying out the $x$ probes sequentially will be more effective than carrying out the $x$ multiple probes simultaneously. In other words, the probes should be carried out one at a time, each time exactly at the point when the variance of a prediction based on the previous estimate is about to exceed the threshold.

\subsection{What if two types of positioning devices are available?}

From the foregoing discussions, we can determine the duration before the next probe is carried out. We will present a min-cost variance-maintaining strategy with two types of devices.

Let $\sigma$ be a required threshold level of the positional variance. Let $\sigma_{a}$ (resp. $\sigma_{b}$ ) denote the standard deviation of an estimate from measurement offered by type-A (resp. type-B) device, where $\sigma_{a}<\sigma_{b}<\sigma$. Let $c$ and 1 denote the cost for each type-A and type-B probe respectively, where $c>1$.

Given a duration of time $T$, we will find the minimum-cost probing sequence by determining the number of type-A probes and type-B probes required over $T$.

Lemma 9: Any probing strategy with the minimum cost is equivalent to a probing sequence in the following two forms:

$$
A \ldots A B \ldots B \text {, }
$$

or

$$
B \ldots B A \ldots A \text {. }
$$

Proof. Each of the probes in a min-cost probing sequence must extend a maximum safe duration, i.e. a probe will be carried out exactly at the point when the variance of a predictive estimate reaches the threshold. Assume that a total of $d$ probes is required. Then the first $d-1$ probes can be permuted in any order, because the overall safe duration is still the same. This completes the proof. 
Let $\tau_{a}$ denote the duration that one type-A probe can sustain until the standard deviation again degrades to the threshold $\sigma$, and, correspondingly, $\tau_{b}$ for type-B. Let $N_{1}=\left\lceil T / \tau_{a}\right\rceil$, and $N_{2}=\left\lceil T / \tau_{b}\right\rceil$.

Lemma 10: A minimum-cost probing sequence can be obtained by comparing at most $N_{1}+N_{2}$ candidate probing sequences.

Proof. Suppose that the best strategy is in the form $A \ldots A B \ldots B$ in which there are $n_{1}$ As and $n_{2}$ Bs. Obviously,

$$
0 \leq n_{1} \leq N_{1}, 0 \leq n_{2} \leq N_{2}
$$

In fact, when given a value of $n_{1}, n_{2}$ is given by $n_{2}=\left\lceil\frac{T-n_{1} \tau_{a}}{\tau_{b}}\right\rceil$.

So the total cost

$$
C\left(n_{1}\right)=c n_{1}+n_{2}=c n_{1}+\left\lceil\frac{T-n_{1} \tau_{a}}{\tau_{b}}\right\rceil
$$

The min-cost sequence $\left(n_{1}^{*}, n_{2}^{*}\right)$ is the one with minimal cost, i.e.

$$
C\left(n_{1}^{*}\right)=\min _{n_{1}} C\left(n_{1}\right),
$$

which can be determined by evaluating each value of $n_{1}$, where $1 \leq n_{1} \leq N_{1}$. A min-cost probing strategy in the form $B \ldots B A \ldots A$ can be determined by examining $N_{2}$ cost values. A min-cost sequence can thus be determined by comparing a total of $N_{1}+N_{2}$ sequences, as claimed.

For example, let $c=2.4, \tau_{a}=2.2, \tau_{b}=1.0, T=15.3$. Then by calculating cost for each pair $\left(n_{1}, n_{2}\right)$, it shows that the minimum cost 15.8 is achieved when $n_{1}=2$, i.e. via the strategy $A A B \ldots(11 B s) \ldots B$.

Theorem 11: Let $\sigma$ denote the standard deviation of the initial estimate. The minimum-cost strategy can be determined by comparing at most $\min \left(N_{1}, N_{2}\right)$ candidate strategies.

Proof. Let $n_{1}, n_{2}$ denote the number of type-A and type-B probes respectively. Then the total cost is $c n_{1}+n_{2}$. We will prove that the minimum-cost can be achieved by strategies in both forms as claimed in Lemma 9 .

Case 1: The optimal probing sequence is in the form $A \ldots A B \ldots B$. In this case, we can check the pair $\left(n_{1}, n_{2}\right)$, where $n_{1}=0,1, \ldots, N_{1}$ and $n_{2}=\left\lceil\frac{T-n_{1} \tau_{a}}{\tau_{b}}\right\rceil$, to obtain the best strategy $\left(i_{1}^{*}, j_{1}^{*}\right)$. Let $C_{1}^{*}$ denote the resulting minimum cost.

Case 2: The optimal probing sequence is in the form $B \ldots B A \ldots A$. In this case, we can verify the pair $\left(n_{1}, n_{2}\right)$, where $n_{2}=0,1, \ldots, N_{2}$ and $n_{1}=\left\lceil\frac{T-n_{2} \tau_{b}}{\tau_{a}}\right\rceil$, to obtain the best strategy $\left(i_{2}^{*}, j_{2}^{*}\right)$. Let $C_{2}^{*}$ denote the resulting minimum cost.

We will show that $C_{1}^{*}=C_{2}^{*}$.

From case 1 we have

$$
j_{1}^{*}=\left\lceil\frac{T-i_{1}^{*} \tau_{a}}{\tau_{b}}\right\rceil,
$$

which implies that

$$
\frac{T-\tau_{a} i_{1}^{*}}{\tau_{b}} \leq j_{1}^{*}<\frac{T-\tau_{a} i_{1}^{*}}{\tau_{b}}+1 .
$$


It can be rewritten as

$$
i_{1}^{*}-\frac{\tau_{b}}{\tau_{a}}<\frac{T-\tau_{b} j_{1}^{*}}{\tau_{a}} \leq i_{1}^{*}
$$

which implies that

$$
\left\lceil\frac{T-\tau_{b} j_{1}^{*}}{\tau_{a}}\right\rceil \leq i_{1}^{*}
$$

Let $\hat{i}=\left\lceil\frac{T-j_{1}^{*} \tau_{b}}{\tau_{a}}\right\rceil$, and $\hat{j}=\left\lceil\frac{T-\tau_{a} \hat{i}}{\tau_{b}}\right\rceil$. Then

$$
\hat{i} \leq i_{1}^{*} \text {. }
$$

The strategy $(\hat{i}, \hat{j})$ is one in the form of case 1 . Let $C_{1}(\hat{i}, \hat{j})$ denote its cost. Then

$$
C_{1}(\hat{i}, \hat{j}) \leq C_{1}^{*} .
$$

From the definition of $\hat{i}$, we have $\tau_{a} \hat{i}+\tau_{b} j_{1}^{*} \geq T$, which results in

$$
\begin{gathered}
j_{1}^{*} \geq \frac{T-\tau_{a} \hat{i}}{\tau_{b}}, \\
j_{1}^{*} \geq \hat{j} .
\end{gathered}
$$

As a result, it must be true that $\hat{i}=i_{1}^{*}$. Otherwise, if $\hat{i}<i_{1}^{*}$, then we have

$$
C_{1}(\hat{i}, \hat{j})<C_{1}^{*},
$$

which means that, in case $1,\left(i_{1}^{*}, j_{1}^{*}\right)$ is not the minimum-cost strategy. So we have proved $\hat{i}=i_{1}^{*}$, which implies $\left(i_{1}^{*}, j_{1}^{*}\right)=\left(\hat{i}, j_{1}^{*}\right)$ is a feasible strategy solution for case 2 , resulting in

$$
C_{1}^{*} \geq C_{2}^{*}
$$

With a similar argument, we have

$$
C_{2}^{*} \geq C_{1}^{*}
$$

We conclude that

$$
C_{1}^{*}=C_{2}^{*} .
$$

The equality shows that the minimum-cost strategy can be found in either cases, which completes the proof.

Remarks Given a journey and two specific types of probes, our method can easily determine a minimum-cost probing sequence. Because our model of the user allows for uncertainties in his move, a probe will generally yield a position measurement that differs from the prediction. If this occurs, we may adaptively recompute the best probing plan based on the latest estimate. 


\section{Related work}

Basically personal positioning consists of two levels of understanding of mobile user's location. One is geographical positioning which provides accurate position information such as GPS latitude-longitude. Another is logical positioning which identifies meaningful landmarks (i.e., significant locations [1]), cell IDs or IP addresses etc. Among those methods which support positionting services, GPS is probably the most developed one and can attain the location within $3 \sim 5$ meter resolution[5]. However, GPS does not work well for indoor or urban environment. As an alternative, cell-based mobile positioning is becoming popular $[4,14,11]$. When in the coverage area of cell tower, a mobile phone can receive/send some information including the cell identification. Consequently, a mobile user's location may also be inferred from the cell probes though at much lower resolution.

When heterogeneous position measurements are available, some data fusion techniques are applied in order to create a good location inference. In [10], the concept of hybrid predictor is proposed in order to use different methods in parallel to improve the estimates. They use prediction models based on neural networks, Bayesian model, and Markov model, and apply three hybrid predictors: the warm-up predictor, the majority predictor, and the confidence predictor. In their experiments, the hybrid predictors shows a better prediction performance than the average prediction of the individual basis methods. However, their approach treats the positioning data obtained from different sources as mathematically-incoherent entities. Another widely-used technique to combine multiple measurements is Kalman filtering [6]. Specifically [9] discusses the application of Kalman filtering on robot localization problems. The robot's location is estimated by using several sensors which output location measurements. For personal positioning, [7] proposes a method that integrates image data from portable sensors by using Kalman filter.

\section{Conclusions}

We have presented a positioning inference method that can uniformly combine multiple sources of positioning information for personal positioning. We have also presented strategies for minimizing the probing cost while controlling the variances of positioning estimates over a journey.

We envision that the method presented will be of use to personal positioning, and hence it will be of great interest to evaluate it on real devices. A natural extension of our current result may be to derive a power-efficient probing strategy for multiple types of probing devices and verify the proposed algorithms on real devices. 


\section{References}

1. D. Ashbrook and T. Starner. Learning significant locations and predicting user movement with gps. International Symposium on Wearable Computing, pages 101$108,2002$.

2. A. Bhattacharya and S. K. Das. Lezi-update: An information-theoretic approach to track mobile users in pcs networks. In Mobile Computing and Networking, pages $1-12,1999$.

3. K.L. Chung. Elementary Probability Theory with Stochastic Processes. SpringerVerlag, New York, NY, 1974.

4. T. D'Roza and G. Bilchev. An overview of location-based services. BT Technology Journal, 21(1):20-27, January 2003.

5. B. Hoffmann-Wellenhof, H. Lichtenegger, and J. Collins. GPS: Theory and Practice. The 3rd ed. Springer-Verlag, New York, 1994.

6. R.E. KALMAN. A new approach to linear filtering and prediction problems. Transactions of the ASME - Journal of Basic Engineering, 82:35-45, 1960.

7. M. Kourogi and T. Kurata. Personal positioning based on walking locomotion analysis with self-contained sensors and a wearable camera. In Proc. of IEEE International Conference on Multisensor Fusion and Integration for Intelligent Systems, MFI'03, pages 287-292, July 2003.

8. T. Manesis and N. Avouris. Survey of position location techniques in mobile systems. In Proceedings of the 7th international conference on Human computer interaction with mobile devices and services, volume 111, pages 291-294, Salzburg, Austria, 2005. ISBN:1-59593-089-2.

9. R. Negenborn. Robot localization and kalman filters- on finding your position in a noisy world. Master's thesis, Institute of Information and Computing Sciences, Utrecht University, September 2003. http://www.negenborn.net/kal_loc/thesis.pdf.

10. J. Petzold, F. Bagci, W. Trumler, and T. Ungerer. Hybrid predictors for next location prediction. the 3rd International Conference on Ubiquitous Intelligence and Computing (UIC-06), pages 125-134, September 2006.

11. A. Roy, S. Das, and K. Basu. A predictive framework for location-aware resource management in smart homes. IEEE Transactions on Mobile Computing, 6(11):1270-1283, November 2007.

12. L. Rudolph, H. Fang, and W.J. Hsu. Adaptive learning/prediction of mobile user's location via historical records of gps and cell id information. Technical Report NTUTR-07-01, Singapore-MIT Alliance of Nanyang Technological University, Singapore, June 2007. Also available as http://www.mit.edu/ fanghui/loc/kfpredict.pdf.

13. Z. Salcic and E. Chan. Mobile station positioning using gsm cellular phone and artificial neural networks. Wireless Personal Communications, 14(3):235-254, September 2000 .

14. E. Trevisani and A. Vitaletti. Cell-id location technique, limits and benefits: An experimental study. In Proceedings of the Sixth IEEE Workshop on Mobile Computing Systems and Applications (WMCSA'04), pages 51-60, 2004.

15. G. Welch and G. Bishop. An introduction to the kalman filter. http://www.cs.unc.edu/ ${ }^{\text {welch. }}$ 